\title{
MicroRNA-655-3p functions as a tumor suppressor by regulating ADAM10 and $\beta$-catenin pathway in Hepatocellular Carcinoma
}

\author{
Gang $\mathrm{Wu}^{1 *}$, Kunming Zheng ${ }^{1}$, Shuguan $\mathrm{Xia}^{2}$, Yawei Wang ${ }^{1}$, Xiangyu Meng ${ }^{3}$, Xiaoming Qin $^{1}$ and Ying Cheng ${ }^{1}$
}

\begin{abstract}
Background: Increasing evidence suggests that microRNAs (miRNAs) play critical roles in malignant transformation, tumor progression and metastasis. Aberrant miR-655-3p expression has been associated with several cancers. However, the role and underlying mechanism of miR-655-3p in the development of hepatocellular carcinoma (HCC) remains unclear.

Methods: MiR-655-3p expression was detected by quantitative RT-PCR (qRT-PCR) in human HCC tissues and cell lines. Cell proliferation was investigated using MTT and colony formation assays, and cell migration and invasion abilities were evaluated by transwell assay. ADAM10 protein expression was detected by immunohistochemical assay. The target gene and downstream of miR-655-3p were determined by qRT-PCR, western blot and dual-luciferase reporter assays.

Results: miR-655-3p was significantly down-regulated in HCC tissues and HCC cell lines. Low miR-655-3p expression was negatively related to tumor size, portal vein tumor thrombosis (PVTT) status, TNM stage and metastasis status. In addition, miR-655-3p overexpression and depletion decreased and increased HCC cell proliferation, migration and invasion, respectively. Moreover, ADAM10 was identified as a direct target of miR-655-3p, and miR-655-3p down-regulated E-cadherin protein level and inhibits $\beta$-catenin pathway by mediating ADAM10.

Conclusions: MiR-655-3p might functions as a tumor suppressor by directly targeting ADAM10 and indirectly regulating $\beta$-catenin pathway in the development of progression of HCC. It may be a novel therapeutic candidate target to in HCC treatment.
\end{abstract}

Keywords: miR-655-3p, Hepatocellular carcinoma, ADAM10, $\beta$-catenin pathway, Proliferation, Migration and invasion

\section{Background}

Hepatocellular carcinoma (HCC), accounting for 85$90 \%$ of primary liver cancers, is the fifth most frequent malignancy and the second leading cause of cancerrelated death in developing countries $[1,2]$. There are many risk factors related to $\mathrm{HCC}$, especially hepatitis B and $C$ viruses and liver cirrhosis [3]. Despite increasing significant advances in surgical techniques and diagnostic methods in recent years, the long-time survival is still

\footnotetext{
* Correspondence: wgzwl@hotmail.com

'Department of General Surgery, The First Affiliated Hospital of China

Medical University, Shenyang 110001, China

Full list of author information is available at the end of the article
}

unsatisfactory mainly due to the high rate of recurrence, metastasis, and emergence of new primary tumors in post operation $[4,5]$. Unfortunately, the mechanisms of high rate of recurrence and metastasis in HCC remain unclear. Therefore, it is urgent to identify the new molecular biomarkers in predicting the aggressive biology of $\mathrm{HCC}$ and guiding effective treatment for HCC patients.

MicroRNAs (miRNAs) are 18-24 nucleotides, small, single-stranded, non-coding RNAs that regulate gene expression by directly degrading mRNA or suppressing post-transcriptional protein translation usually by binding to the 3' untranslated region (3' -UTR) of the respective target mRNAs [6]. It has been confirmed that 
ample miRNAs can function as tumor suppressors or oncogenes to play important roles in the initiation, promotion and progression of various cancers [7], and aberrant miRNAs expression might be of potential use as a diagnostic and prognostic biomarker for human cancer including HCC. For example, miR-20, miR-182, miR-494, and miR-125b have been proved to regulate tumor cell growth, migration and invasion in HCC [8-11].

There are several miRNAs encoded in $14 \mathrm{q} 32$ locus, including miR-655-3p, miR-127-5p, miR-369-3p, miR544a. MiRNAs on this locus have been reported associated with a metastatic phenotype in clinical cancer samples [12], and ectopic expression of 14q32-encoded microRNAs can reduce cell-autonomous metastatic properties in vitro and inhibit metastasis development in vivo [13]. MiR-655-3p expression is reduced in several cancers and overexpression of miR-655 act as a tumor suppressor by targeting pituitary tumor-transforming gene-1(PTTG1) in esophageal squamous cell carcinoma metastasis [14]. Previous study has demonstrated that miR-655inhibits epithelial mesenchymal transition (EMT) suppressive miRNA by targeting regulate ZEB1 and TGFBR2 inducing inactivation of the TGF- $\beta$ signaling pathway [15]. In addition, miR-134/487b/655 cluster is also reported to regulate TGF- $\beta$ induced EMT and gefitinib resistance by targeting MAGI2 in lung adenocarcinoma cells [16]. However, the expression level of miR-655-3p and its roles in the development of HCC have not yet been reported.

In the current study, we investigated the biological function and molecular mechanism of miR-655-3p in HCC. MiR-655-3p was significantly decreased in HCC clinical specimens and cell lines. Overexpression of miR-655-3p suppressed cell proliferation, migration and invasion of HCC in vitro. Further study showed that miR-655-3p could down-regulate E-cadherin and inhibit $\beta$-catenin pathway by targeting $\mathrm{A}$ Disintegrin and Metalloprotease Domain 10(ADAM10). Our findings elucidated the detailed roles of miR-655-3p in HCC and further contribute to offering the effective therapeutic targets for the treatment of HCC.

\section{Methods}

\section{Patients and specimens}

Primary tumor tissues and their corresponding adjacent non-tumorous liver specimens were obtained from 84 patients who were diagnosed with HCC during hepatic resection in the First Affiliated Hospital of China Medical University between July 2013 and July 2014. None had received preoperative radiotherapy or chemotherapy before surgery. Fresh specimens were snap-frozen and stored in liquid nitrogen tanks immediately after resection. The clinical and pathological parameters are shown in Table 1. This study protocol was approved by the
Table 1 miR-655-3p expression and clinicopathological features in hepatocellular carcinoma patients

\begin{tabular}{|c|c|c|c|c|}
\hline \multirow[t]{2}{*}{ Characteristics } & \multicolumn{3}{|c|}{ miR-655-3p expression } & \multirow[t]{2}{*}{$p$-value } \\
\hline & Cases & Low & middle/High & \\
\hline \multicolumn{5}{|l|}{ Age (years) } \\
\hline$\geq 53$ & 43 & $27(62.8 \%)$ & $16(37.2 \%)$ & \multirow[t]{2}{*}{0.69} \\
\hline$<53$ & 41 & $24(58.5 \%)$ & $17(41.5 \%)$ & \\
\hline \multicolumn{5}{|l|}{ Gender } \\
\hline Male & 71 & $45(63.4 \%)$ & $26(36.6 \%)$ & \multirow[t]{2}{*}{0.242} \\
\hline Female & 13 & $6(46.2 \%)$ & $7(53.8 \%)$ & \\
\hline \multicolumn{5}{|l|}{ HBsAg status } \\
\hline Positive & 62 & $36(58.1 \%)$ & $26(41.9 \%)$ & \multirow[t]{2}{*}{0.404} \\
\hline Negative & 22 & $15(68.2 \%)$ & $7(31.8 \%)$ & \\
\hline \multicolumn{5}{|l|}{ AFP (ng/ml) } \\
\hline$\geq 20$ & 48 & $30(62.5 \%)$ & $18(37.5 \%)$ & \multirow[t]{2}{*}{0.699} \\
\hline$<20$ & 36 & $21(58.3 \%)$ & $15(41.7 \%)$ & \\
\hline \multicolumn{5}{|l|}{ Liver cirrhosis } \\
\hline Yes & 63 & $38(60.3 \%)$ & $25(49.7 \%)$ & \multirow[t]{2}{*}{0.897} \\
\hline No & 21 & $13(61.9 \%)$ & $8(39.1 \%)$ & \\
\hline \multicolumn{5}{|c|}{ Tumour size $(\mathrm{cm})$} \\
\hline$<5$ & 44 & $22(50 \%)$ & $22(50 \%)$ & \multirow[t]{2}{*}{$0.035^{*}$} \\
\hline$\geq 5$ & 40 & $29(72.5 \%)$ & $11(27.5 \%)$ & \\
\hline \multicolumn{5}{|l|}{ PVTT } \\
\hline Yes & 9 & $9(100.0 \%)$ & $0(0.0 \%)$ & \multirow[t]{2}{*}{$0.028^{*}$} \\
\hline No & 75 & $42(56 \%)$ & $33(44 \%)$ & \\
\hline \multicolumn{5}{|c|}{ TNM Classification } \\
\hline$|+| \mid$ & 50 & $24(48 \%)$ & $26(52 \%)$ & \multirow[t]{2}{*}{$0.004^{* *}$} \\
\hline$I I I+I V$ & 34 & $27(79.4 \%)$ & $7(20.6 \%)$ & \\
\hline \multicolumn{5}{|c|}{ Tumour differentiation } \\
\hline Well & 37 & $21(56.8 \%)$ & $16(43.2 \%)$ & \multirow[t]{3}{*}{0.721} \\
\hline Moderate & 34 & $21(61.8 \%)$ & $13(48.2 \%)$ & \\
\hline Poor & 13 & $9(69.2 \%)$ & $4(30.8 \%)$ & \\
\hline \multicolumn{5}{|l|}{ Metastasis } \\
\hline Yes & 33 & $27(81.8 \%)$ & $6(18.2 \%)$ & \multirow[t]{2}{*}{$0.001^{* *}$} \\
\hline No & 51 & 24(47.1\%) & $27(52.9 \%)$ & \\
\hline
\end{tabular}

Abbreviations: AFP alpha-fetoprotein, $H B s A g$ hepatitis B surface antigen, TNM tumor-node-metastasis, PVTT portal vein tumor thrombosis. ${ }^{*} \mathrm{p}<0.05 ;{ }^{* *} \mathrm{p}<0.01$

Institutional Ethics Committee of China Medical University, and human tumor tissues for this research were obtained with informed consent.

\section{HCC cell lines and cell cultures}

Seven HCC cell lines, HepG2, SK-hep1, HCCLM3, Huh7, MHCC-97H, MHCC-97 L, BEL-7402, and one normal liver cell line, LO2, were used in this study. The Huh7, BEL-7402, SK-hep1, HCCLM3 and LO2 human cell lines were obtained from the Institute of Biochemistry and Cell Biology at the Chinese Academy of 
Sciences (Shanghai, China). The HepG2, MHCC97H and MHCC97L cells were obtained from Chinese Academy of Medical Sciences (Beijing, China). HCCLM3, Huh7, HepG2, MHCC97H, MHCC97L were cultured in DMEM medium. BEL-7402, SK-Hep1, LO2 were cultured in RPMI 1640 medium. All the medium was added with $10 \%$ fetal bovine serum (FBS) and 100 units/ml of penicillin and streptomycin (Hyclone, USA). All cells were grown in a humidified incubator with $5 \% \mathrm{CO} 2$ at $37^{\circ} \mathrm{C}$.

\section{$R N A$ preparation and quantitative real-time $P C R$}

Total RNA from HCC tissue samples and adjacent nontumorous tissue samples was extracted using Trizol Reagent (Invitrogen, USA) according to the manufacturer's instructions. To determine mature miRNA expression levels, qRT-PCR was performed using a SYBR Premix Ex Taq (TaKaRa, Japan) on a Thermal Cycler Dice Real Time System (TaKaRa) with the following protocol: $30 \mathrm{~s}$ at $95{ }^{\circ} \mathrm{C}$ followed by two-step PCR for 40 cycles of $95{ }^{\circ} \mathrm{C}$ for $5 \mathrm{~s}$ and $60{ }^{\circ} \mathrm{C}$ for $60 \mathrm{~s}$. MiRNA expression levels were normalized against the endogenous U6 small nuclear RNA (U6 snRNA) control. ADAM10 expression was measured by SYBR green qPCR assay and GAPDH was used as an endogenous control. The relative expression level of miR-655-3p in each paired tumor and adjacent non-tumorous tissue was calculated by the $2^{-\Delta \Delta C T}$ method. The sequences of the PCR primers were as follows: miR-655-3p forward, 5'-CCGCGATAATACATG GTTAACCTC-3,' and reverse primer was Uni-miR qPCR primer (TaKaRa); U6 forward, 5'-CTCGCTTCGGCAGCACA-3' and U6 reverse, 5'-AACGCTTCACGAATTT GCGT-3'; ADAM10 forward, 5'-CTGCCCAGCATCTGACCCTAA-3' and reverse, 5'-TTGCCATCAGAACTG GCACAC-3'; GAPDH forward, 5'CTCCTCCTGTTCGA CAGTCAGC-3', and reverse 5'-CCCAATACGACCAAA TCCGTT-3'.

\section{Oligonucleotides transfection}

The miR-655-3p agomiR (agomiR-655-3p), antagomiR (anti-miR-655-3p), small interfering RNA for ADAM10 (siADAM10) and their negative control (Neg.Cont) Oligonucleotides used in this study were purchased from Shanghai GenePharma Co. Ltd. Transfection was performed using Lipofectamine 2000 (Invitrogen) according to the manufacturer's protocol. The sequences of Oligonucleotides were as follows: agomiR-655-3p, sense 5'AUAAUACAUGGUUAACCUCUUU-3' and antisense 5'AGAGGUUAACCAUGUAUUAUUU-3'; miRNA negative controls, sense 5'-UUCUCCGAACGUGUCACGUTT-3' and antisense 5'-ACGUGACACGUUCGGAGAATT-3'; anti-miR-655-3p, 5'-AAAGAGGUUAACCAUGUAUUAU
-3'; negative control, 5'-UUGUACUACACAAAAGUACU G-3'; Si-ADAM10, 5'-CAGUGUGCAUUCAAGUCAA-3'.

\section{Luciferase assay}

The wild-type ADAM10-3'UTR(WT) and mutant ADA M10-3'UTR(MUT) containing the putative binding site of miR-655-3p were established and cloned in the Firefly luciferase expressing vector pMIR-REPORT (Obio Technology, China). Liver cancer cells were seeded into 24-well plates the day before transfection, and transfected with either the pMIR-REPORT-ADAM10-3' UTR-WT or the PMIR-REPORT-ADAM10-3' UTRMUT reporter vector, together with the Renilla luciferase-expressing vector pRL-TK (Promega) and agomiR-655-3p or miR-Neg.Cont using Lipofectamine 2000 (Invitrogen). After 48 h, cells were harvested, and firefly and Renilla luciferase activities were measured using the dual-luciferase reporter assay system (Promega, Madison, WI).

\section{Cell migration and invasion assays}

After $48 \mathrm{~h}$ of transfection, cell concentration in each group was adjusted to $2 \times 10^{5}$ cells $/ \mathrm{mL}$ with serum-free medium. The upper chamber of Transwell chamber (Costar; 24-well insert, pore size: $8 \mu \mathrm{m}$ ) was filled with $200 \mu \mathrm{l}$ cell suspension, and the lower chamber was filled with $500 \mu \mathrm{L}$ of medium supplementing $15 \%$ FBS. For the invasion assay, polycarbonate filters coated with $50 \mu \mathrm{L}$ Matrigel (1:9, BD Bioscience) were placed in a Transwell chamber. Three wells were used for each group. Cells were incubated for $24 \mathrm{~h}$ for the migration assay and $48 \mathrm{~h}$ for the invasion assay. Then, the cells on the upper surface were wiped slightly using cotton swabs, and the cells on the lower surface were fixed with $4 \%$ paraformaldehyde and stained with $0.1 \%$ crystal violet. The migratory cells were visualized and counted in five random visual fields per insert under an inverted microscope at 200× magnification (Nikon MicrophotFX, Japan).

\section{MTT assay}

After transfection, 5000 cells/well were seeded in 96well plates in media containing $10 \% \mathrm{FBS}$ and incubated for $0,24 \mathrm{~h}, 48 \mathrm{~h}, 72 \mathrm{~h}$. On the indicated days, 3-(4,5)dimethylthiahiazo(-z-y1)-3,5-di-phenytetrazoliumromide (MTT) (KyeGEN BioTECH, Nanjing, China) was added into each well according to the manufacturer's instructions, and the cells were incubated for $4 \mathrm{~h}$ at $37^{\circ} \mathrm{C}$. The supernatants were then removed and $150 \mathrm{uL}$ of DMSO (Sigma-Aldrich, Germany) was added to per well to dissolve the formazan crystals. Absorbance levels were measured at the wavelength of $490 \mathrm{~nm}$ using an automatic microplate reader (Gene, HK). The data derived from triplicate samples are presented as mean \pm s.d. 


\section{Colony formation assay}

After transfection, 500 cells per well were counted and seeded in 6-well plates. The plates were incubated for 10 days, then the cells were fixed by $4 \%$ paraformaldehyde and stained using $0.1 \%$ crystal violet. Colonies were counted only if they included 50 cells at least. Triplicate independent experiments were performed and all the visible colonies were calculated manually.

\section{Western blot}

Cell samples were washed with ice-cold PBS and then lysed by RIPA (Beyotime, China) containing protease inhibitors (Beyotime, China). Cell protein lysates were separated in $10 \%$ SDS-PAGE and then transferred onto a polyvinylidene difluoride (PVDF) membrane (Millipore, USA). The membranes were blocked by $5 \%$ skim milk soluted in TBST buffers, and were incubated with primary antibodies for ADAM10, c-myc (Abcam, UK), E-cadherin, MMP9 (Santa Cruz Biotechnology, USA), $\beta$-catenin and cyclinD1 (ProteinTech Group, USA) overnight at $4{ }^{\circ} \mathrm{C}$. PVDF membranes were washed in TBST and incubated with horseradish peroxidaseconjugated secondary antibodies (ProteinTech Group, USA). Antibody against GAPDH (Cell Signaling Technology, USA) was used as an internal control. Antibody against Histone H3 (Abcam, UK) was used as an internal control for nuclear $\beta$-catenin. The protein of interest was visualized using ECL Western blotting substrate (Pierce, USA).

\section{Immunohistochemical (IHC)}

Formalin-stabilised liver tissue specimens were embedded in paraffin and cut into $4 \mu \mathrm{m}$ sections for use in immunohistochemistry. After general deparaffinization, antigen retrieval was carried out for $30 \mathrm{sec}$ with an autoclave using $0.01 \mathrm{~mol} / \mathrm{l}$ sodium citrate buffer, $\mathrm{pH}$ 6.0. $\mathrm{H} 2 \mathrm{O} 2(0.3 \%)$ was used to block endogenous peroxidase activity for $30 \mathrm{~min}$ at $37{ }^{\circ} \mathrm{C}$, and nonspecific immunoglobulin binding sites were blocked by normal goat serum for $30 \mathrm{~min}$ at $37{ }^{\circ} \mathrm{C}$. Sections were then incubated overnight with primary antibody(ADAM10,1:300, Abcam) at $4{ }^{\circ} \mathrm{C}$, rinsed with PBS, and incubated with the appropriate secondary antibody for $30 \mathrm{~min}$. The peroxidase reaction was developed with 3 , 3-diaminobenzidine tetrahydrochloride (DAB).Sections were counterstained with Mayer's hematoxylin, dehydrated, cleared in xylene, and mounted in Permount.

\section{Statistical analysis}

The statistical analyses were performed using the SPSS 17.0 software. Parametric data were presented as mean \pm SEM, and differences between each group were analyzed using the Student's t-test. The association between miR$655-3 p$ relative expression and the clinicopathological parameters was evaluated by the $\mathrm{X} 2$ test or Fisher's exact test when appropriate. All of the $\mathrm{p}$-values reported were two-sided, and significance was defined as $p<0.05$.

\section{Result Analysis \\ MiR-655-3p expression in HCC tissues and cell lines}

To analysis the miRNA-655-3p expression pattern, 84 pairs of HCC tissues and adjacent non-tumorous liver tissues were detected by qRT-PCR. The result showed that down-regulation of miR-655-3p was observed in 61 (72.6\%) cases of HCC tissues, which was significantly lower than that in matched non-tumorous tissues $(P$ $<0.001$, Fig. 1a, b). In cell level, miR-655-3p expression was lower in the HCCLM3, HepG2, SK-hep1, MHCC97H, Huh7, MHCC-97 L cell lines than that in the normal liver cell line LO2 (Fig. 1c). All the above results indicated that miR-655-3p was down-regulated in HCC.

\section{Association of miR-655-3p expression with clinicopathological features}

In order to explore the potential clinical significance of miR-655-3p in HCC patients, the cases were divided into miR-655-3p low-expression group $(n=51)$ and mid/ high-expression group $(n=33)$, according to the relative ratio of miR-655-3p expression in tumor/adjacent nontumor $<$ or $>0.5$. The correlation between miR-655-3p expression and clinicopathological characteristics was shown in Table 1. MiR-655-3p expression was positively associated with tumor size $(p=0.035)$, PVTT $(p=0.028)$, TNM stage $(p=0.004)$ and metastasis $(p=0.001)$, respectively. However, it was no correlations with gender, age, preoperative serum AFP and histological differentiation. Based on these findings, we speculated miR-6553 p might play a vital role in HCC development.

\section{Ectopic expression of miR-655-3p inhibits HCC cell lines proliferation}

To examine the functional roles of miR-655-3p in HCC, we upregulated HCCLM3 and HepG2 cells by miR-655$3 \mathrm{p}$ agomiR (100nM) transfection. Overexpression of miR-655-3p in the two HCC cell lines were confirmed by qRT-PCR after transfection for $48 \mathrm{~h}$ (Fig. 2a, b). Then MTT and colony formation assays were performed to detected proliferation ability. Compared to the negative control group, the cancer cell proliferation was dramatically inhibited in miR-655-3p overexpression group by MTT analysis after transfection for $48 \mathrm{~h}$ and $72 \mathrm{~h}$ (Fig. 2d, e). Consistent with the MTT assay, colony formation assay also showed that miR-655-3p overexpression led to a significant reduction of colony number in HCC cells (Fig. 2g, h). Conversely, miR-655-3p inhibitor significantly promoted the proliferation potential in Huh7 cells both in MTT and colony formation assays 


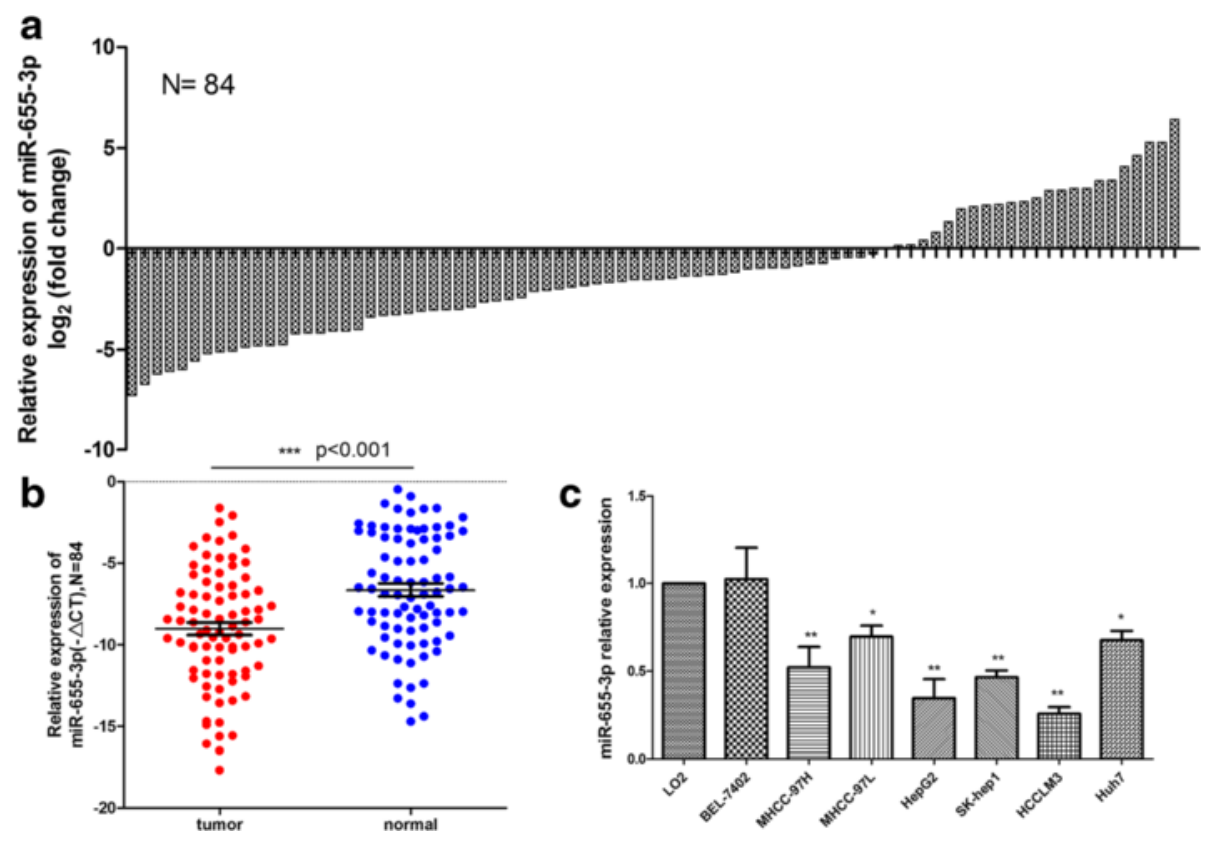

Fig. 1 MiR-655-3p is low-expressed in HCC tissues and cell lines. a QRT-PCR analysis of miR-655-3p expression in 84 pairs HCC and their corresponding adjacent nontumorous livers tissues. The expression of miRNA was normalized to U6 snRNA. $\mathbf{b}$ Relative miR-655-3p expression levels in HCC tissues and adjacent normal regions; c QRT-PCR analysis of miR-655-3p expression in HCC cells (Bel-7402, MHCC-97 L, MHCC-97H, HepG2, SK-Hep1, HCCLM3, Huh7) and normal hepatocytes (LO-2)

(Fig. 2c, f, i). These results proved that miR-655-3p inhibit proliferation in HCC.

\section{Restoration of miR-655-3p represses migration and invasion of HCC cells}

To investigate the function of miR-655-3p in cell migration and invasion, transwell chamber assay was performed in HCC cells. We found enhancement of the expression of miR-655-3p in HepG2 and HCCLM3 cells could significantly inhibit cell invasion and migration abilities. The number of invasive and migrated cells in the miR-655-3p overexpression group $(82 \pm 5$ and $58 \pm 6$, respectively) was significantly decreased, compared with the negative control group (180 \pm 8 and $105 \pm 7$, respectively) in HepG2 cells. The same results were also observed in HCCLM3 cells ( $97 \pm 8$ and $87 \pm 8$ vs. $212 \pm 24$ and $116 \pm 10$, respectively). Conversely, anti-agomiR$655-3 p$ significantly increased the cell migration and invasion of the Huh7 cells $(202 \pm 10$ and $182 \pm 8$ vs. $92 \pm 6$ and 79 \pm 6 ) (Fig. 3). Based on these results, we concluded that miR-655-3p decreased the migration and invasion of $\mathrm{HCC}$ cells.

\section{MiR-655-3p can upregulate E-cadherin expression and inhibit $\beta$-catenin signal pathway in $\mathrm{HCC}$ cells}

To better understand the underlying molecular mechanism of miR-655-3p inhibiting proliferation and metastasis of HCC, we detected the expression of potential proliferation and metastasis-associated proteins by western blot. The results showed that miR-655-3p upregulation in HCCLM3 and HepG2 cells increased Ecadherin and decreased the nucleus $\beta$-catenin (the total $\beta$-catenin showed no change), cyclinD1, c-myc protein levels(Fig. 4a, b, c). Conversely, inhibiting miR655-3p in Huh7 cells decreased E-cadherin and unregulated nucleus $\beta$-catenin, cyclinD1 and $c$-myc proteins (Fig. 4a, d). Only nucleus $\beta$-catenin decrease after miR-655-3p overexpression indicated that miR655-3p may affect the distribution of $\beta$-catenin. All these results suggested that miR-655-3p might influence the biological behavior of HCC by regulating E-cadherin expression and inhibiting $\beta$-catenin signal pathway.

\section{ADAM10 is a direct target of miR-655-3p in HCC}

It has been demonstrated miRNA relies on its regulating target genes to achieve its biological function [17]. Therefore, we identified miR-655 target genes using the target prediction tool, miRwalk, a comprehensive database on miRNAs with six established program (miRanda, miRDB, miRWalk, TargetScan, RNA22, and PITA) [18]. There were no miR-655-3p binding sites predicted on mRNA of E-cadherin, $\beta$-catenin, cyclinD1 and c-myc. While, ADAM10 was one of the gene that was predicted to binding miR-655 by at least five program and related to HCC biological progress according to the relevant previous reports $[19,20]$. 


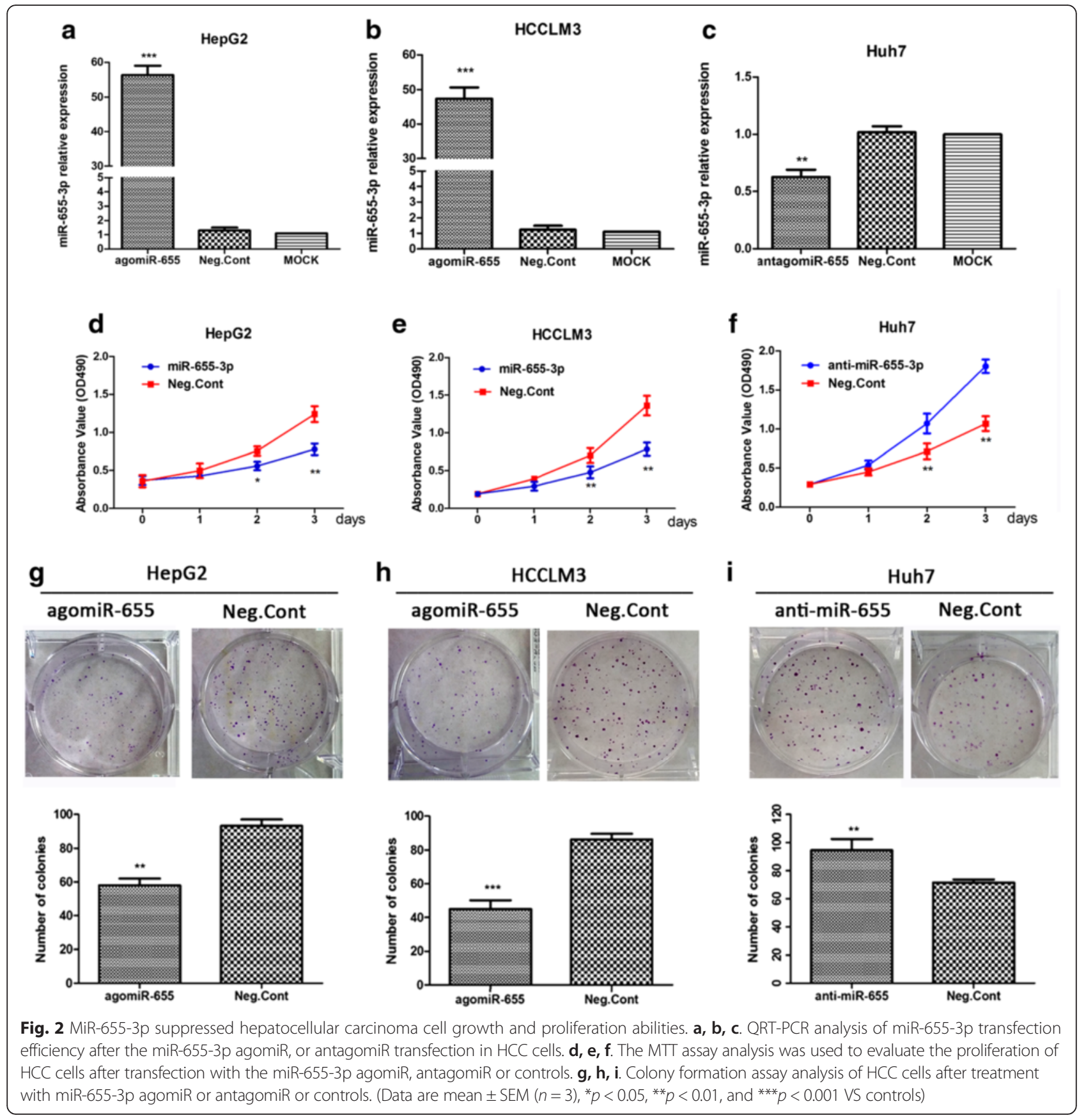

As predicted, overexpression of miR-655-3p in HepG2 and HCCLM3 cells decreased the expression of ADAM10 at both the mRNA and protein levels, whereas miR-655-3p inhibitor increased its expression in Huh7 cells (Fig. 5a, b, d). Using TargetScan, we located potential binding sites for miR-655-3p at the 3'UTR of ADAM10 mRNAs (Fig. 5c). Then, a dual-luciferase reporter system was carried out to determine whether ADAM10 was a direct target of miR-655-3p. Overexpression of miR-655-3p significantly suppressed the luciferase activity of the wild-type ADAM10 3'-UTR, but failed to affect the mutant 3'-UTR in HCCLM3 and HepG2 cells (Fig. 5e, f). Taken together, these results demonstrated that ADAM10 was a direct target of miR-655-3p.

\section{Loss-of function of ADAM10 mimicked impact of} miR-655-3p on HCC cell proliferation and metastasis

To confirm whether miR-655-3p dependent repression of HCC cell biological behaviors was mediated by ADAM10, we investigated the expression and role of ADAM10 in HCC. ICH results showed that expression 

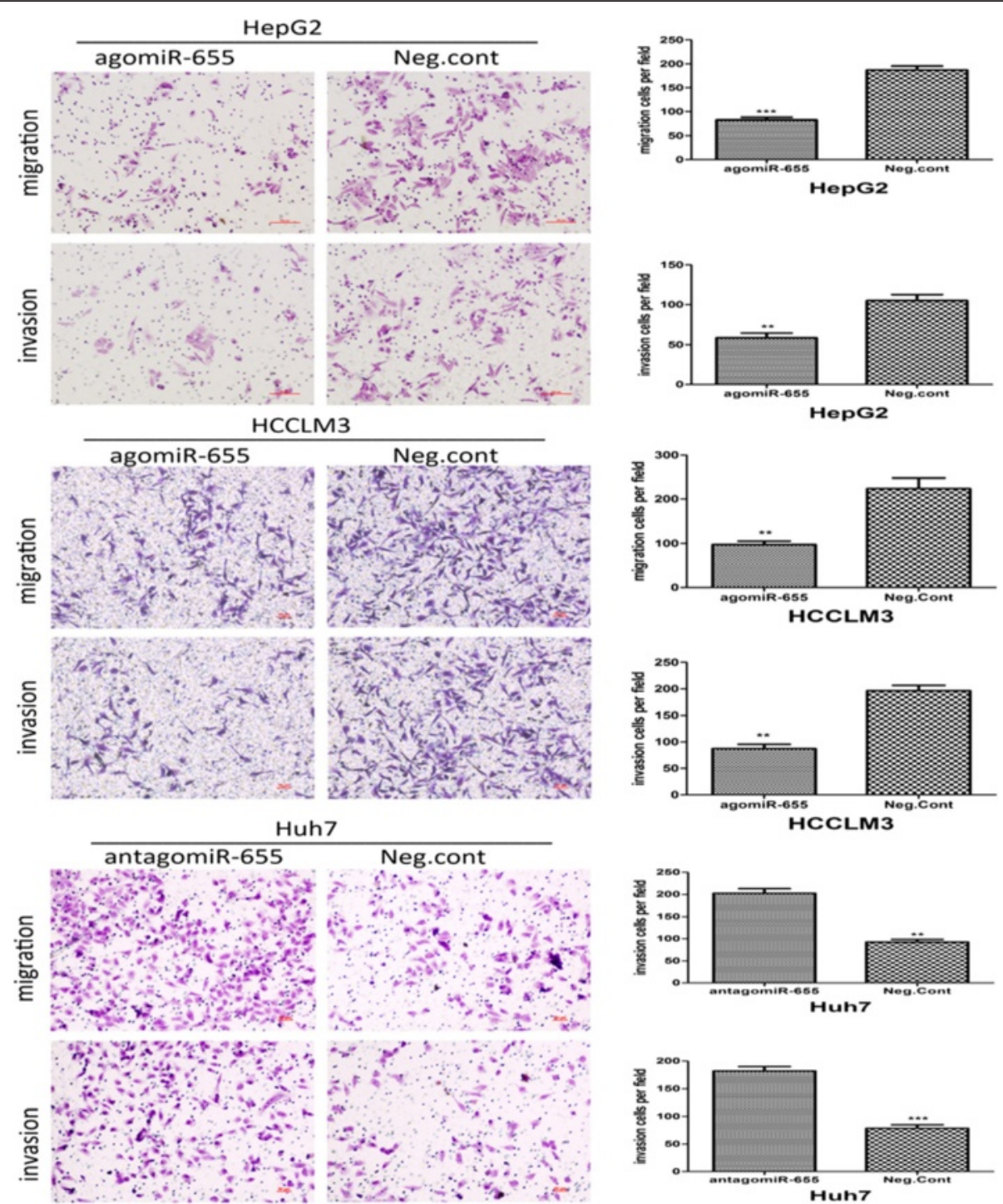

Fig. 3 Effect of miR-655-3p on invasion and migration in HCC. Restoration of miR-655-3p repressed migration and invasion of HepG2 and HCCLM3 cells; and inhibiting miR-655-3p expression promoted cell migration and invasion in Huh7 cells. Transwell analysis was used. Data are mean $\pm \operatorname{SEM}(n=3) .{ }^{*} p<0.05,{ }^{* *} p<0.01$, and ${ }^{* * *} p<0.001$ compared to controls

of ADAM10 is strongly positively stained in HCC tissues, while, absent or sporadic in non-tumorous liver tissues. And ADAM10 is located in the cytoplasm and cell membrane in HCC ADAM10-positive cells (Fig. 6a, b). The further results revealed that si-ADAM10 mediated downregulation of ADAM10 expression inhibited the proliferation, migration and invasion both in HepG2 and Huh7 cells (Fig. 6d, e, f, g). The effect of ADAM10 silencing was similar to the effect of miR-655-3p overexpression on proliferation, migration and invasion of HepG2 cells (Fig. 2d, Fig. 3). Moreover, inhibition of ADAM10 expression significantly attenuated the abilities of cell proliferation, migration and invasion promoted by anti-miR-655-3p in Huh7 cells (Fig. 6c, h). These results demonstrated that miR-655-3p inhibited the proliferation, migration and invasion of HCC cells by targeting ADAM10.

\section{Discussion}

Tumor invasion, metastatic dissemination, recurrence, and drug resistance have been identified as the major causes of the poor clinical outcome in HCC patients [1-4]. More and more miRNAs were converged to account for characters of various tumor processes, including tumor initiation, development and metastasis $[6,21]$. MiR-655-3p was encoded in 14q32 locus, a region that has been implicated in oncogenesis and metastasis of diverse cancer types [12, 13]. Yang et al. showed miR-655 expression was decreased in esophageal squamous cell carcinoma (ESCC) and overexpression of miR- 


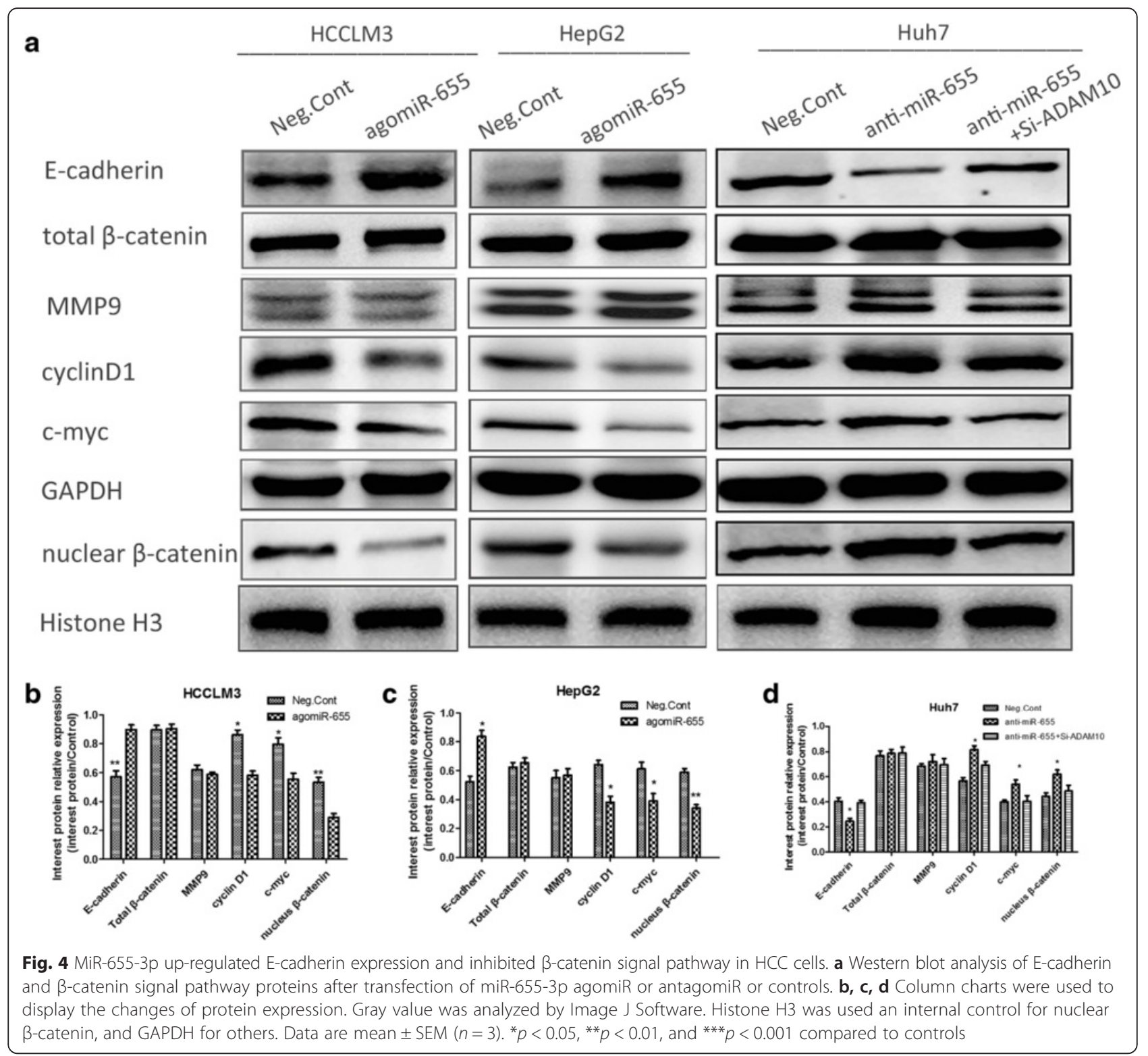

655 inhibited ESCC cell invasiveness by targeting PTTG1 [14]. Yosuke et al. reported that miR-655 represses EMT progress through inducing inactivation of the TGF- $\beta$ signaling pathway by targeting ZEB1 and TGFBR2, in ESCC [15]. Kitamura et al. demonstrated that miR-134/487b/655 cluster regulate TGF- $\beta$ induced EMT and drug resistance to Gefitinib by targeting MAGI2 in lung adenocarcinoma cells [16]. In addation, Zhang et al. showed that Norcantharidin suppress glioblastoma cell invasion through modulation of miR-655mediated SUMO-specific protease 6 translation [22]. Various publications have associated the dysregulation of miR-655 with cancer progress. However, the relationship between miR-655 and HCC remains unknown.
In this study, we first demonstrated that miR-655-3p was significantly down-regulated in human HCC tissues and cell lines. We also found that down-regulation of miR-655-3p expression levels was significantly associated with the tumor size, portal vein tumor thrombosis(PVTT) status, TNM stage and metastasis status. Using MTT, colony formation and transwell assays, we found that overexpression of miR-655-3p could suppress the proliferation, migration and invasion ability in HCC cells in vitro, indicating the crucial role of miR-655-3p in HCC development. Based on above results, miR-655$3 \mathrm{p}$ might was a tumor suppressor in HCC. Then, the underlying molecular mechanism of miR-655-3p inhibiting $\mathrm{HCC}$ progress was detected, and the results showed 


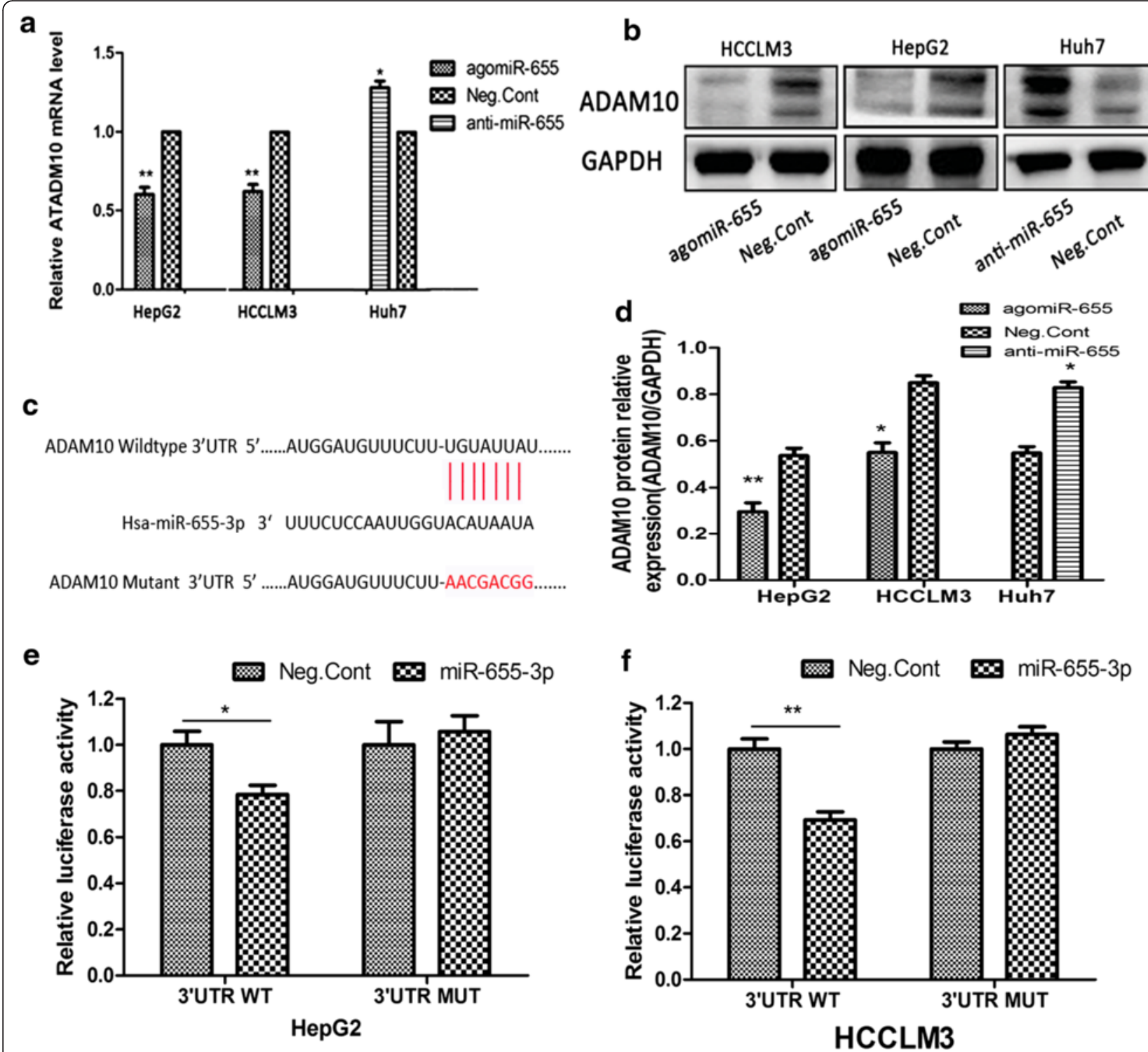

Fig. 5 ADAM10 is a direct target of miR-655-3p in HCC. a. qRT-PCR analysis of ADAM10 mRNA expression after the miR-655-3p agomiR, or antagomiR transfection in HCC cells. $\mathbf{b}$, $\mathbf{d}$. Western blot analysis of ADAM10 protein expression after the miR-655-3p agomiR, or antagomiR transfection in HCC cells; and their Column charts analysis. c. The predicted interaction site of miR-655-3p and candidate target gene ADAM10 wild-type 3'-UTR and serial deleted forms of the 3'UTR reporters. e, f. Luciferase assay of co-transfected with miR-655-3p agomiR and pMIRREPORT-ADAM10 plasmid (miR-NC and miR-655-3p with ADAM10 WT 3'UTR; miR-NC and miR-655-3p with ADAM10 MUT $3^{\prime} U T R$ ) after $24 \mathrm{~h}$. Data are mean $\pm \operatorname{SEM}(n=3) .{ }^{*} p<0.05$, and ${ }^{* *} p<0.01$ compared to controls

upregulating miR-655-3p expression increased E-cadherin protein but decreased the nucleus $\beta$-catenin, cyclinD1 and c-myc expression. $\beta$-catenin is known to interact with TCF/LEF family of transcription factors to induce gene expression, such as cyclinD1 and c-myc [21, 23-25]. These results indicate that miR-655-3p might influence the biological behavior of HCC by regulating E-cadherin expression and inhibiting $\beta$-catenin signal pathway.

A disintegrin and metalloproteinase 10 (ADAM10), has been proved to be upregulated in various cancers and involved in cancer progression and metastasis, such as pancreatic cancer, breast cancer, lung cancer and hepatocellular carcinoma [26-30]. ADAM10 is reported as a sheddase that can cleave transmembrane proteins such as amyloid precursor protein (APP), E-cadherin, $\mathrm{N}$-cadherin, CD44 and Notch, all of which play a significant role in proliferation, migration, invasion or stemness of cancer cells [27, 28, 31-35]. Of particular importance is that ADAM10 is identified as an oncogene contributing to $\mathrm{HCC}$ progression, such as metastasis, invasion and drug resistance of Sorafenib [18, 19]. In our study, we also found that ADAM10 silencing 


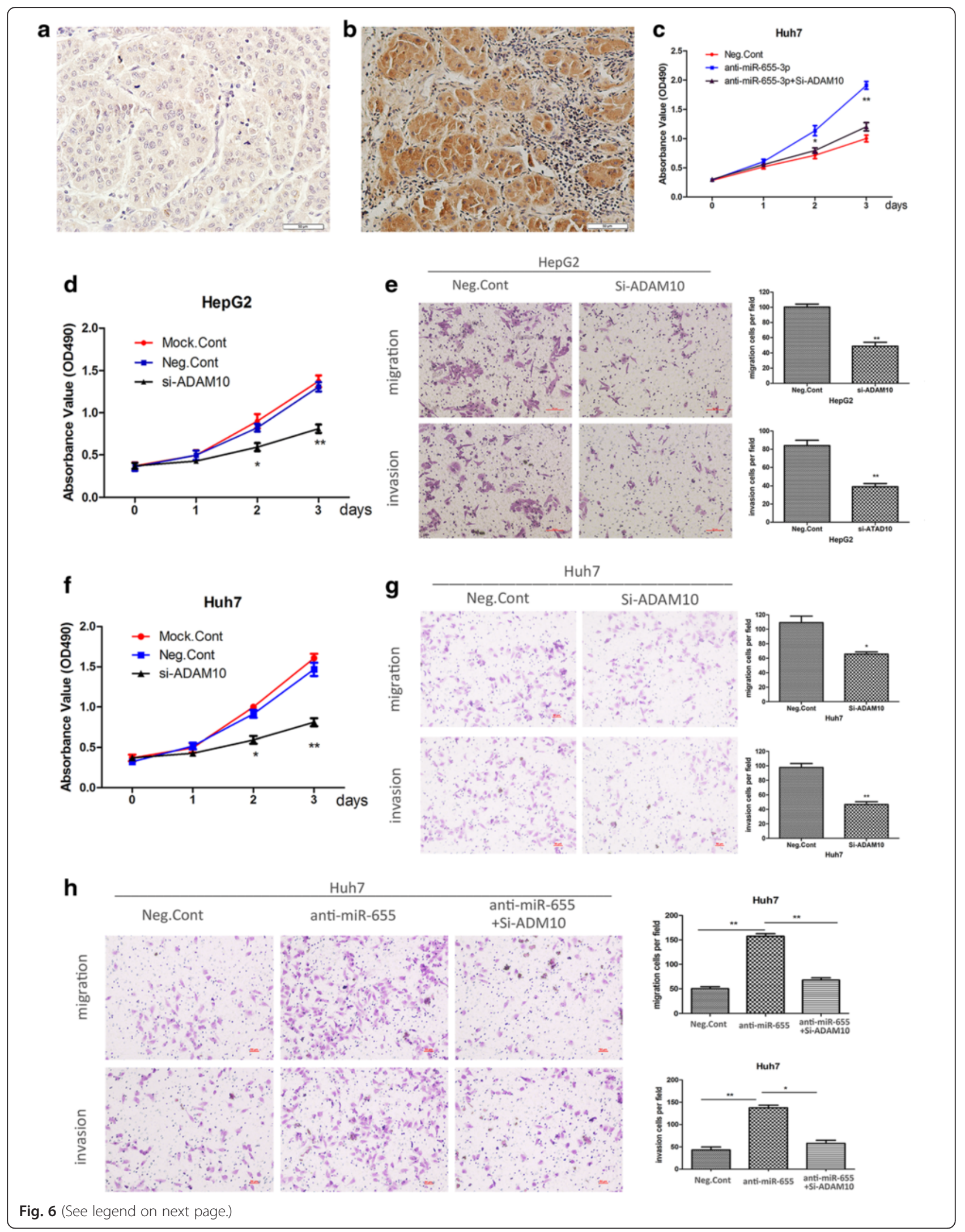


(See figure on previous page.)

Fig. 6 Loss-of function of ADAM10 mimicked impact of miR-655-3p on HCC cell proliferation and metastasis. $\mathbf{a}$, $\mathbf{b}$ IHC staining of ADAM10 in HCC and normal liver tissues. d, $\mathbf{f}$ The MTT assay analysis of proliferation after Si-ADAM10 or controls transfected in HepG2 and Huh7 cells respectively. e, $\mathbf{g}$ Transwell analysis was used to detect the impact of migration and invasion after Si-ADAM10 or controls transfected in HepG2 and Huh7 cells respectively. c The MTT assay analysis of proliferation ability of Huh7 cells after transfected miR-655-3p antagomiR or co-transfected Si-ADAM10 or controls. $\mathbf{h}$ Transwell analysis of migration and invasion abilities of Huh7 cells after transfected miR-655-3p antagomiR or co-transfected Si-ADAM10 or controls. Data are mean $\pm \operatorname{SEM}(n=3)$. ${ }^{*} p<0.05$, and ${ }^{* *} p<0.01$ compared to controls

suppresses HCC proliferation, migration and invasion in vitro, and using a luciferase-based reporter assay, we demonstrated that miR-655-3p could bind to a sequence within the 3'-UTR of ADAM10. MiR-655-3p-mediated control of ADAM10 expression was further validated by complementary gain and loss-of-function approaches. Thus, we conclude that miR-655-3p mediates HCC progress by targeting the 3'-UTR of ADAM10. Neha et al. reported that inhibiting ADAM10 expression subsequently diminishes $\beta$-catenin intracellular signaling and repress TCF/LEF target gene expression [27], which is similar to our founding that miR-655-3p could reduce $E$ cadherin protein level and inhibit $\beta$-catenin pathway by target regulating ADAM10 in HCC. Besides, ADAM10 can exert different functions regulated by multiple micrornas. For example, miR-144/451 inhibits cancer metastasis by targeting ADAMTS5 and ADAM10 in human epithelial cancers [36]; miR-122-5p reduces trastuzumab resistance by regulating ADAM10 in breast cancer [37]; miR-140-5p can repress tumor progression by targeting ADAM10 in human tongue and hypopharyngeal squamous cancer cells [38, 39]. Our study provided a new mechanism accounting for ADAM10 dysregulation in HCC.

\section{Conclusion}

In conclusion, our study demonstrate that miR-655-3p functions as tumor suppressor by directly targeting ADAM10 and indirectly regulating $\beta$-catenin pathway in $\mathrm{HCC}$ progression and metastasis. These findings provide a new insight into the molecular pathogenesis of $\mathrm{HCC}$ and identify miR-655-3p as a novel therapeutic candidate target for HCC.

\footnotetext{
Abbreviations

3'-UTR, 3' untranslated region; ADAM10, A Disintegrin and Metalloprotease Domain; EMT, epithelial mesenchymal transition; ESCC, esophageal squamous cell carcinoma; HCC, Hepatocellular carcinoma; miRNAs, microRNAs; MTT, 3-(4,5)-dimethylthiahiazo(-z-y1)-3,5-di-phenytetrazoliumromide; PVTT, portal vein tumor thrombosis; qRT-PCR, real time polymerase chain reaction; TCF/LEF, T-cell factor and Lymphoid enhancing factor
}

\section{Acknowledgements}

This work was supported by a grant from Population and Health Research Projects from Shenyang Science and Technology Bureau (No.F15-139-9-25), Science and Technology Committee of Liaoning Province (No.2015020529), and Fund for Scientific Research of the First Hospital of China Medical University (FSFH1511).

\section{Authors' contributions}

GW designed experiments. KMZ, SGX and GW conducted all experiments. GW and KMZ wrote the manuscript. GW, KMZ, SGX, XYM, YWW, XMQ and YC contributed to data analysis and discussed the results. All authors read and approved the final manuscript.

\section{Competing interests}

The authors declare that they have no competing interests.

\section{Author details}

${ }^{1}$ Department of General Surgery, The First Affiliated Hospital of China Medical University, Shenyang 110001, China. ${ }^{2}$ Department of Breast and Thyroid Surgery, Huaihe Hospital, Henan University, Kaifeng 475000, China. ${ }^{3}$ Department of Gastric Surgery, Liaoning Cancer Hospital and Institute, Shenyang 110001, China.

Received: 17 March 2016 Accepted: 1 June 2016

Published online: 04 June 2016

\section{References}

1. Siegel R, Ma J, Zou Z, Jemal A. Cancer statistics, 2014. CA Cancer J Clin. 2014;64:9-29.

2. Singal AG, El-Serag HB. Hepatocellular Carcinoma From Epidemiology to Prevention: Translating Knowledge into Practice. Clin Gastroenterol Hepatol. 2015;13:2140-51.

3. El-Serag HB, Rudolph KL. Hepatocellular carcinoma: epidemiology and molecular carcinogenesis. Gastroenterology. 2007;132:2557-76.

4. Graf D, Vallbohmer D, Knoefel WT, Kropil P, Antoch G, Sagir A, Haussinger D. Multimodal treatment of hepatocellular carcinoma. Eur J Intern Med. 2014:25:430-7.

5. Schwartz M, Roayaie S, Konstadoulakis M. Strategies for the management of hepatocellular carcinoma. Nat Clin Pract Oncol. 2007;4:424-32.

6. Falcone G, Felsani A, D'Agnano I. Signaling by exosomal microRNAs in cancer. J Exp Clin Cancer Res. 2015;34:32.

7. Macfarlane LA, Murphy PR. MicroRNA: Biogenesis, Function and Role in Cancer. Curr Genomics. 2010;11:537-61.

8. Fan MQ, Huang CB, Gu Y, Xiao Y, Sheng JX, Zhong L. Decrease expression of microRNA-20a promotes cancer cell proliferation and predicts poor survival of hepatocellular carcinoma. J Exp Clin Cancer Res. 2013;32:21.

9. Zhou JN, Zeng Q, Wang HY, Zhang B, Li ST, Nan X, Cao N, Fu CJ, Yan XL, Jia $Y L$, et al. MicroRNA-125b attenuates epithelial-mesenchymal transitions and targets stem-like liver cancer cells through small mothers against decapentaplegic 2 and 4. Hepatology. 2015;62:801-15.

10. Du C, Weng X, Hu W, et al. Hypoxia-inducible MiR-182 promotes angiogenesis by targeting RASA1 in hepatocellular carcinoma. J Exp Clin Cancer Res. 2015;34:67.

11. Chuang KH, Whitney-Miller CL, Chu CY, Zhou Z, Dokus MK, Schmit S, Barry CT. MicroRNA-494 is a master epigenetic regulator of multiple invasionsuppressor microRNAs by targeting ten eleven translocation 1 in invasive human hepatocellular carcinoma tumors. Hepatology. 2015;62:466-80.

12. Vanharanta S, Massague J. Origins of metastatic traits. Cancer Cell. 2013;24:410-21.

13. Uppal A, Wightman SC, Mallon S, Oshima G, Pitroda SP, Zhang Q, Huang X, Darga TE, Huang L, Andrade J, et al. 14q32-encoded microRNAs mediate an oligometastatic phenotype. Oncotarget. 2015;6:3540-52.

14. Wang Y, Zang W, Du Y, Ma Y, Li M, Li P, Chen X, Wang T, Dong Z, Zhao G. Mir-655 up-regulation suppresses cell invasion by targeting pituitary tumortransforming gene-1 in esophageal squamous cell carcinoma. J Transl Med. 2013;11:301

15. Harazono Y, Muramatsu T, Endo H, Uzawa N, Kawano T, Harada K, Inazawa J, Kozaki K. miR-655 Is an EMT-suppressive microRNA targeting ZEB1 and TGFBR2. PLoS One. 2013;8:e62757. 
16. Kitamura K, Seike M, Okano T, Matsuda K, Miyanaga A, Mizutani H, Noro R, Minegishi Y, Kubota K, Gemma A. MiR-134/487b/655 cluster regulates TGFbeta-induced epithelial-mesenchymal transition and drug resistance to gefitinib by targeting MAGI2 in lung adenocarcinoma cells. Mol Cancer Ther. 2014;13:444-53.

17. Giordano S, Columbano A. MicroRNAs: new tools for diagnosis, prognosis, and therapy in hepatocellular carcinoma? Hepatology. 2013;57:840-7.

18. Dweep H, Sticht C, Pandey P, Gretz N. miRWalk-database: prediction of possible miRNA binding sites by "walking" the genes of three genomes. J Biomed Inform. 2011;44:839-47.

19. Zhang W, Liu S, Liu K, Wang Y, Ji B, Zhang X, Liu Y. A disintegrin and metalloprotease (ADAM) 10 is highly expressed in hepatocellular carcinoma and is associated with tumour progression. J Int Med Res. 2014;42:611-8.

20. Yuan S, Lei S, Wu S. ADAM10 is overexpressed in human hepatocellular carcinoma and contributes to the proliferation, invasion and migration of HepG2 cells. Oncol Rep. 2013;30:1715-22.

21. Zhang $X$, Hao J. Development of anticancer agents targeting the Wnt/betacatenin signaling. Am J Cancer Res. 2015;5:2344-60.

22. Zhang $Z$, Song $X$, Feng $X$, Miao $Y$, Wang H, Li Y, Tian H. Norcantharidin modulates miR-655-regulated SENP6 protein translation to suppresses invasion of glioblastoma cells. Tumour Biol. 2015 Nov 25. [Epub ahead of print]

23. Novellasdemunt L, Antas P, Li VS. Targeting Wnt signaling in colorectal cancer. A Review in the Theme: Cell Signaling: Proteins, Pathways and Mechanisms. Am J Physiol Cell Physiol. 2015;309:C511-21.

24. Blagodatski A, Poteryaev D, Katanaev VL. Targeting the Wnt pathways for therapies. Mol Cell Ther. 2014;2:28.

25. Kanwar SS, Yu Y, Nautiyal J, Patel BB, Majumdar AP. The Wnt/beta-catenin pathway regulates growth and maintenance of colonospheres. Mol Cancer. 2010;9:212.

26. You B, Shan Y, Shi S, Li X, You Y. Effects of ADAM10 upregulation on progression, migration, and prognosis of nasopharyngeal carcinoma. Cancer Sci. 2015;106(11):1506-14.

27. Woods N, Trevino J, Coppola D, Chellappan S, Yang S, Padmanabhan J. Fendiline inhibits proliferation and invasion of pancreatic cancer cells by interfering with ADAM10 activation and beta-catenin signaling. Oncotarget. 2015;6:35931-48.

28. Shitomi Y, Thogersen IB, Ito N, Leitinger B, Enghild JJ, Itoh Y. ADAM10 controls collagen signaling and cell migration on collagen by shedding the ectodomain of discoidin domain receptor 1 (DDR1). Mol Biol Cell. 2015;26:659-73.

29. Chang YC, Jan Cl, Peng CY, Lai YC, Hu FW, Yu CC. Activation of microRNA494-targeting Bmi1 and ADAM10 by silibinin ablates cancer stemness and predicts favourable prognostic value in head and neck squamous cell carcinomas. Oncotarget. 2015;6:24002-16.

30. Feldinger K, Generali D, Kramer-Marek G, Gijsen M, Ng TB, Wong JH, Strina C, Cappelletti M, Andreis D, Li JL, et al. ADAM10 mediates trastuzumab resistance and is correlated with survival in HER2 positive breast cancer. Oncotarget. 2014;5:6633-46.

31. Maretzky T, Evers A, Le Gall S, Alabi RO, Speck N, Reiss K, Blobel CP. The cytoplasmic domain of a disintegrin and metalloproteinase 10 (ADAM10) regulates its constitutive activity but is dispensable for stimulated ADAM10dependent shedding. J Biol Chem. 2015;290:7416-25.

32. Nakamura H, Suenaga N, Taniwaki K, Matsuki H, Yonezawa K, Fujii M, Okada Y, Seiki M. Constitutive and induced CD44 shedding by ADAM-like proteases and membrane-type 1 matrix metalloproteinase. Cancer Res. 2004;64:876-82

33. van Tetering $G$, van Diest $P$, Verlaan I, van der Wall E, Kopan R, Vooijs M. Metalloprotease ADAM10 is required for Notch1 site 2 cleavage. J Biol Chem. 2009;284:31018-27.

34. Stamenkovic I, Yu Q. Shedding light on proteolytic cleavage of CD44: the responsible sheddase and functional significance of shedding. J Invest Dermatol. 2009;129:1321-4

35. Grabowska MM, Sandhu B, Day ML. EGF promotes the shedding of soluble E-cadherin in an ADAM10-dependent manner in prostate epithelial cells. Cell Signal. 2012;24:532-8.

36. Zhang J, Qin X, Sun Q, Guo H, Wu X, Xie F, Xu Q, Yan M, Liu J, Han Z, Chen W. Transcriptional control of PAX4-regulated miR-144/451 modulates metastasis by suppressing ADAMs expression. Oncogene. 2015;34:3283-95.

37. Ergun $S$, Ulasli M, Igci $Y Z$, Igci M, Kirkbes S, Borazan E, Balik A, Yumrutas O, Camci C, Cakmak EA, et al. The association of the expression of miR-122-5p and its target ADAM10 with human breast cancer. Mol Biol Rep. 2015;42:497-505.
38. Jing P, Sa N, Liu X, Liu X, Xu W. MicroR-140-5p suppresses tumor cell migration and invasion by targeting ADAM10-mediated Notch1 signaling pathway in hypopharyngeal squamous cell carcinoma. Exp Mol Pathol. 2015;100:132-8.

39. Kai $Y$, Peng W, Ling W, Jiebing $H$, Zhuan B. Reciprocal effects between microRNA-140-5p and ADAM10 suppress migration and invasion of human tongue cancer cells. Biochem Biophys Res Commun. 2014;448:308-14.

\section{Submit your next manuscript to BioMed Central and we will help you at every step:}

- We accept pre-submission inquiries

- Our selector tool helps you to find the most relevant journal

- We provide round the clock customer support

- Convenient online submission

- Thorough peer review

- Inclusion in PubMed and all major indexing services

- Maximum visibility for your research

Submit your manuscript at www.biomedcentral.com/submit
Biomed Central 